\title{
Adjudication process
}

Each of the following modes of death could be marked as primary, secondary on noncontributory: sudden death, terminal heart failure, stroke, cardiac procedure, other cardiovascular (such as myocardial infarction, pulmonary embolism, and aortic aneurysm rupture), cancer, infection or other non-cardiovascular. For each patient, only one primary mode can be selected, and multiple secondary modes as appropriate. Cause of death is deemed cardiovascular if the primary mode was sudden death, terminal heart failure, stroke, cardiac procedure or other cardiovascular. All other cases, including deaths due to infection or cancer, were adjudicated as “non-cardiovascular”.

For in-patient death, we used information reported by the last treating physician on the death certificate in conjunction with diagnostic tests and blood results available in the days leading up to death. All deaths in the emergency department were considered sudden deaths, unless investigations or correspondence suggested differently.

For out-patient deaths, if a patient had recently been seen in clinic or discharged from hospital without warning of a poor prognosis, end-of-life planning or significant abnormality on investigations and then died without other healthcare contact, the mode of death was defined as sudden. Similarly, if a patient died outside hospital and there was no evidence of severe infection or terminal illness (including cancer, heart failure and advanced dementia) on the electronic records, the primary mode of death was also considered to be sudden.

If a patient had recurrent hospital admissions with acute heart failure, or was persistently in New York Heart Association (NYHA) class III or IV in clinic in the months prior to death, then the primary mode was terminal heart failure. Similarly, if the patient had been referred 
to palliative care or long-term care facility after a hospitalisation due to terminal illness such as cancer or heart failure then that illness was adjudicated to be the mode of death.

Where sufficient information was lacking to adjudicate death (for instance because the patient moved out of the region) the mode and cause of death were adjudicated as "unknown”. 


\begin{tabular}{|c|c|c|c|c|c|c|c|}
\hline Variable & Missing & $\begin{array}{l}\text { No HF } \\
n=667\end{array}$ & $\begin{array}{c}\text { HFrEF } \\
n=1408\end{array}$ & $\begin{array}{c}\text { HFmrEF } \\
n=786\end{array}$ & $\begin{array}{c}\text { HFpEF } \\
n=1562\end{array}$ & $P$ all groups & $\begin{array}{c}\text { P - } \\
\text { only HF groups } \\
\end{array}$ \\
\hline \multicolumn{8}{|c|}{ Demographics } \\
\hline Age - years & 0 & $66(59-73)$ & $71(63-78)$ & $74(67-80)$ & $76(70-82)$ & $<0.001$ & $<0.001$ \\
\hline Sex (male) - no. (\%) & 0 & $362(54)$ & $1058(75)$ & $534(68)$ & $741(47)$ & $<0.001$ & $<0.001$ \\
\hline Body mass index $-\mathrm{kg} / \mathrm{m}^{2}$ & 28 & $31(6)$ & $28(6)$ & $29(6)$ & $30(6)$ & $<0.001$ & $<0.001$ \\
\hline Systolic blood pressure $-\mathrm{mmHg}$ & 21 & $\begin{array}{c}144 \\
(130-159) \\
\end{array}$ & $\begin{array}{c}126 \\
(112-142) \\
\end{array}$ & $\begin{array}{c}137 \\
(121-157) \\
\end{array}$ & $\begin{array}{c}148 \\
(130-164) \\
\end{array}$ & $<0.001$ & $<0.001$ \\
\hline Heart rate - bpm & 35 & $71(62-82)$ & $74(63-87)$ & $71(60-84)$ & $71(61-83)$ & $<0.001$ & $<0.001$ \\
\hline Sinus rhythm - no. (\%) & 49 & $666(100)$ & $1067(78)$ & $515(66)$ & $1049(67)$ & $<0.001$ & $<0.001$ \\
\hline Peripheral oedema $\geq$ ankles - no. (\%) & 394 & $89(15)$ & $292(23)$ & $212(30)$ & $464(32)$ & $<0.001$ & $<0.001$ \\
\hline Lung crackles - no. (\%) & 666 & $33(6)$ & $215(18)$ & $114(17)$ & $173(13)$ & $<0.001$ & 0.001 \\
\hline Raised JVP - no. (\%) & 631 & $15(3)$ & $229(19)$ & $98(15)$ & $168(13)$ & $<0.001$ & $<0.001$ \\
\hline Diabetes - no. (\%) & 0 & $189(28)$ & $329(23)$ & $195(25)$ & $427(27)$ & 0.030 & 0.043 \\
\hline h/o Hypertension - no. (\%) & 0 & $379(57)$ & $537(38)$ & $405(52)$ & $1019(65)$ & $<0.001$ & $<0.001$ \\
\hline IHD - no. (\%) & 0 & $179(27)$ & $922(66)$ & $464(59)$ & $529(34)$ & $<0.001$ & $<0.001$ \\
\hline Previous or current smokers - no. (\%) & 0 & $113(17)$ & $318(23)$ & $115(15)$ & $178(11)$ & $<0.001$ & $<0.001$ \\
\hline NYHA Class I - no. (\%) & \multirow{4}{*}{18} & $307(46)$ & $189(13)$ & $148(19)$ & $415(27)$ & \multirow{4}{*}{$<0.001$} & \multirow{4}{*}{$<0.001$} \\
\hline NYHA Class II - no. (\%) & & $252(38)$ & $671(48)$ & $387(49)$ & $727(47)$ & & \\
\hline NYHA Class III - no. (\%) & & $96(15)$ & $503(36)$ & $240(31)$ & $394(25)$ & & \\
\hline NYHA Class IV - no. (\%) & & $6(1)$ & $39(3)$ & $10(1)$ & $21(1)$ & & \\
\hline \multicolumn{8}{|c|}{ Blood results } \\
\hline NT-proBNP - ng/L & 220 & $61(38-93)$ & $1811(775-4080)$ & 1023 (449-2326) & $659(271-1505)$ & NA & NA \\
\hline NT-proBNP in SR - ng/l & 158 & $61(38-93)$ & $1372(617-3606)$ & $727(313-1846)$ & $389(211-846)$ & $<0.001$ & $<0.001$ \\
\hline NT-proBNP in AF - ng/l & 57 & NA & $\begin{array}{c}3069 \\
(1755-6085) \\
\end{array}$ & $\begin{array}{c}1687 \\
(936-3378) \\
\end{array}$ & $\begin{array}{c}1499 \\
(926-2562) \\
\end{array}$ & NA & $<0.001$ \\
\hline HsCRP - mg/L & 0 & $2.7(1.3-5.1)$ & $4.2(1.7-9.1)$ & $3.9(1.6-9.0)$ & $3.7(1.5-7.9)$ & $<0.001$ & 0.019 \\
\hline Haemoglobin - g/L & 220 & $14.0(1.4)$ & $13.4(1.7)$ & $13.3(1.8)$ & $13.0(1.7)$ & $<0.001$ & $<0.001$ \\
\hline White cell count $-10^{9} \cdot \mathrm{L}^{-1}$ & 215 & $6.7(5.8-8.2)$ & $7.3(6.1-8.6)$ & $7.3(6.0-8.7)$ & $7.2(5.9-8.5)$ & 0.004 & 0.75 \\
\hline Neutrophils- $10^{9} \cdot \mathrm{L}^{-1}$ & 623 & $3.9(3.2-5.1)$ & $4.6(3.7-5.7)$ & $4.5(3.6-5.7)$ & $4.5(3.6-5.7)$ & 0.57 & 0.31 \\
\hline Lymphocytes $-10^{9} \cdot \mathrm{L}^{-1}$ & 623 & $1.9(1.6-2.4)$ & $1.6(1.2-2.1)$ & $1.7(1.3-2.1)$ & $1.7(1.3-2.1)$ & 0.14 & 0.13 \\
\hline Plasma viscosity - mPaSec & 1147 & $1.7(0.1)$ & $1.7(0.2)$ & $1.7(0.1)$ & $1.7(0.2)$ & $<0.001$ & 0.03 \\
\hline
\end{tabular}




\begin{tabular}{|c|c|c|c|c|c|c|c|}
\hline Creatinine- $\mu \mathrm{mol} / \mathrm{L}$ & 94 & $82(71-97)$ & $105(88-131)$ & $100(84-124)$ & $93(77-117)$ & $<0.001$ & $<0.001$ \\
\hline Urea - mmol/L & 100 & $5.2(4.2-6.3)$ & $7.1(5.4-9.7)$ & $6.7(5.2-9.4)$ & $6.5(5.0-8.7)$ & $<0.001$ & $<0.001$ \\
\hline Albumin - g/L & 106 & $39(3)$ & $38(4)$ & $37(4)$ & $37(4)$ & $<0.001$ & 0.052 \\
\hline Cholesterol -mmol/L & 232 & $4.8(4.0-5.8)$ & $4.4(3.6-5.3)$ & $4.3(3.6-5.3)$ & $4.4(3.7-5.4)$ & $<0.001$ & 0.25 \\
\hline Bilirubin - $\mu \mathrm{mol} / \mathrm{L}$ & 120 & $13(11-16)$ & $15(12-20)$ & $14(11-18)$ & $13(11-17)$ & $<0.001$ & $<0.001$ \\
\hline ALP - iu/L & 108 & $68(57-84)$ & $74(60-92)$ & $74(60-92)$ & $73(59-92)$ & $<0.001$ & 0.94 \\
\hline ALT - iu/L & 111 & $24(19-32)$ & $21(17-28)$ & $20(16-27)$ & $19(16-25)$ & $<0.001$ & $<0.001$ \\
\hline \multicolumn{8}{|c|}{ "Medications } \\
\hline Loop diuretic - no (\%) & 0 & $191(29)$ & $1074(76)$ & $495(63)$ & $820(53)$ & $<0.001$ & $<0.001$ \\
\hline Mineralocorticoid antagonists- no (\%) & 0 & $30(4)$ & $479(34)$ & $166(21)$ & $131(8)$ & $<0.001$ & $<0.001$ \\
\hline ACE-I or ARB- no (\%) & 0 & $309(46)$ & $1147(82)$ & $582(74)$ & $916(59)$ & $<0.001$ & $<0.001$ \\
\hline Beta-blockers- no (\%) & 0 & $188(28)$ & $937(67)$ & $502(64)$ & $772(50)$ & $<0.001$ & $<0.001$ \\
\hline Statins - no (\%) & 0 & $323(48)$ & $803(57)$ & $432(55)$ & $804(52)$ & 0.001 & 0.009 \\
\hline \multicolumn{8}{|c|}{ Echocardiography } \\
\hline 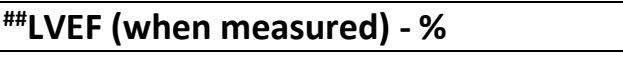 & 1976 & $59(53-63)$ & $30(25-35)$ & $44(42-47)$ & $58(55-63)$ & NA & NA \\
\hline LAD - cm & 498 & $3.6(0.5)$ & $4.3(0.8)$ & $4.3(0.8)$ & $4.1(0.8)$ & $<0.001$ & $<0.001$ \\
\hline
\end{tabular}

Table 1 supplementary: patients characteristics by diagnosis and HF phenotypes. ${ }^{*}$ Medications recorded at baseline prior to changes subsequent to initial referral. ${ }^{\#}$ LVEF was measured in 2,023 patients (54\%) and was visually estimated in the remainder as $<40 \%$, $40-49 \%$ or $\geq 50 \%$. Abbreviations used: JVP - jugular venous pressure; IHD - ischaemic heart disease; HFrEF - heart failure with reduced ejection fraction; HFmrEF - heart failure with midrange ejection fraction; HFpEF - heart failure with preserved ejection fraction; SR - sinus rhythm; AF - atrial fibrillation; ALT - alanine aminotransferase; ALP - alkaline phosphatase; LVEF - left ventricular ejection fraction; LAD - left atrial diameter. 


\begin{tabular}{|c|c|c|c|c|c|}
\hline \multicolumn{6}{|c|}{ Mortality at 2 years in patients with HF } \\
\hline & $\begin{array}{c}\text { All } \\
\mathrm{N}=3538 \#\end{array}$ & $\begin{array}{c}\text { HsCRP } \\
<2 \\
\mathrm{mg} / \mathrm{L} \\
\mathbf{N}=1057\end{array}$ & $\begin{array}{c}\text { HsCRP } \\
2.0-4.9 \\
\mathrm{mg} / \mathrm{L} \\
\mathrm{N}=975\end{array}$ & $\begin{array}{c}\text { HsCRP } \\
5.0-9.9 \\
\mathrm{mg} / \mathrm{L} \\
\mathrm{N}=752\end{array}$ & $\begin{array}{c}\text { HsCRP } \\
\geq 10 \\
\mathrm{mg} / \mathrm{L} \\
\mathrm{N}=754\end{array}$ \\
\hline 2 year mortality rate & $\begin{array}{l}\mathbf{1 8 . 9 \%} \\
(n=670)\end{array}$ & $\begin{array}{l}\mathbf{1 1 . 8 \%} \\
(n=125)\end{array}$ & $\begin{array}{l}13.3 \% \\
(n=130)\end{array}$ & $\begin{array}{c}21.1 \% \\
(n=159)\end{array}$ & $\begin{array}{l}33.9 \% \\
(n=256)\end{array}$ \\
\hline \multicolumn{6}{|c|}{ Cause and mode of Death } \\
\hline Cardiovascular & $\begin{array}{c}\mathbf{1 1 . 6 \%} \\
(\mathrm{n}=413)\end{array}$ & $\begin{array}{l}\mathbf{8 . 4 \%} \\
(\mathrm{n}=89)\end{array}$ & $\begin{array}{l}7.9 \% \\
(\mathrm{n}=77)\end{array}$ & $\begin{array}{c}\mathbf{1 3 . 4 \%} \\
(\mathrm{n}=101)\end{array}$ & $\begin{array}{c}\mathbf{1 9 . 3 \%} \\
(\mathrm{n}=146)\end{array}$ \\
\hline Terminal HF & $\begin{array}{l}3.4 \% \\
(n=122)\end{array}$ & $\begin{array}{l}2.4 \% \\
(n=25)\end{array}$ & $\begin{array}{l}2.0 \% \\
(\mathrm{n}=20)\end{array}$ & $\begin{array}{r}4.5 \% \\
(n=34)\end{array}$ & $\begin{array}{c}5.7 \% \\
(\mathrm{n}=43)\end{array}$ \\
\hline Sudden & $\begin{array}{l}\mathbf{6 . 8 \%} \\
(\mathrm{n}=241)\end{array}$ & $\begin{array}{l}5.0 \% \\
(\mathrm{n}=53)\end{array}$ & $\begin{array}{l}\mathbf{4 . 8 \%} \\
(\mathrm{n}=47)\end{array}$ & $\begin{array}{c}7.4 \% \\
(n=56)\end{array}$ & $\begin{array}{c}11.3 \% \\
(n=85)\end{array}$ \\
\hline Other $C V$ & $\begin{array}{l}1.4 \% \\
(n=50)\end{array}$ & $\begin{array}{l}\mathbf{1 . 0 \%} \\
(\mathrm{n}=11)\end{array}$ & $\begin{array}{l}\mathbf{1 . 1 \%} \\
(\mathrm{n}=10)\end{array}$ & $\begin{array}{l}\mathbf{1 . 5 \%} \\
(\mathrm{n}=11)\end{array}$ & $\begin{array}{l}2.3 \% \\
(n=18)\end{array}$ \\
\hline Non-CV & $\begin{array}{l}7.2 \% \\
(n=257)\end{array}$ & $\begin{array}{c}3.5 \% \\
(\mathrm{n}=36)\end{array}$ & $\begin{array}{l}5.4 \% \\
(\mathrm{n}=53)\end{array}$ & $\begin{array}{l}7.7 \% \\
(n=58)\end{array}$ & $\begin{array}{c}\mathbf{1 4 . 6 \%} \\
(\mathrm{n}=110)\end{array}$ \\
\hline Infection & $\begin{array}{l}3.1 \% \\
(n=110)\end{array}$ & $\begin{array}{l}1.3 \% \\
(\mathrm{n}=14)\end{array}$ & $\begin{array}{c}2.7 \% \\
(\mathrm{n}=26)\end{array}$ & $\begin{array}{c}3.2 \% \\
(n=24)\end{array}$ & $\begin{array}{c}\mathbf{6 . 1 \%} \\
(\mathrm{n}=46)\end{array}$ \\
\hline Cancer & $\begin{array}{l}2.7 \% \\
(n=95)\end{array}$ & $\begin{array}{l}\mathbf{1 . 1 \%} \\
(\mathrm{n}=11)\end{array}$ & $\begin{array}{l}\mathbf{1 . 7 \%} \\
(\mathrm{n}=17)\end{array}$ & $\begin{array}{c}2.9 \% \\
(n=22)\end{array}$ & $\begin{array}{l}\mathbf{6 . 0 \%} \\
(\mathrm{n}=45)\end{array}$ \\
\hline Other Non CV/Unknown * & $\begin{array}{l}1.4 \% \\
(\mathrm{n}=52)\end{array}$ & $\begin{array}{l}\mathbf{1 . 1 \%} \\
(\mathrm{n}=11)\end{array}$ & $\begin{array}{l}\mathbf{1 . 0 \%} \\
(\mathrm{n}=10)\end{array}$ & $\begin{array}{l}\mathbf{1 . 6 \%} \\
(\mathrm{n}=12)\end{array}$ & $\begin{array}{l}2.5 \% \\
(\mathrm{n}=19)\end{array}$ \\
\hline \multicolumn{6}{|c|}{ Origin of Cancer Attributed to Death } \\
\hline Lung & 31 & 2 & 7 & 4 & 18 \\
\hline Prostate & 5 & 0 & 2 & 1 & 2 \\
\hline GI & 23 & 6 & 4 & 6 & 7 \\
\hline Blood & 10 & 1 & 0 & 5 & 4 \\
\hline Renal/Urinary & 6 & 0 & 1 & 1 & 4 \\
\hline Breast & 5 & 0 & 0 & 3 & 2 \\
\hline Pancreas & 4 & 2 & 0 & 1 & 1 \\
\hline Liver & 2 & 0 & 0 & 0 & 2 \\
\hline Other/Unknown & 9 & 0 & 3 & 1 & 5 \\
\hline
\end{tabular}

Table 2 supplementary. Rate, cause and mode of death at 2 years in patients with HF, according to different cut-offs of HsCRP. Abbreviations used: CV: cardiovascular; GI: gastrointestinal; HF: heart failure. \# excludes 218 patients who had follow-up shorter than 2 years. * The mode and cause of death were adjudicated as "unknown" in two patients only (one with HFmrEF and one with HFpEF). 


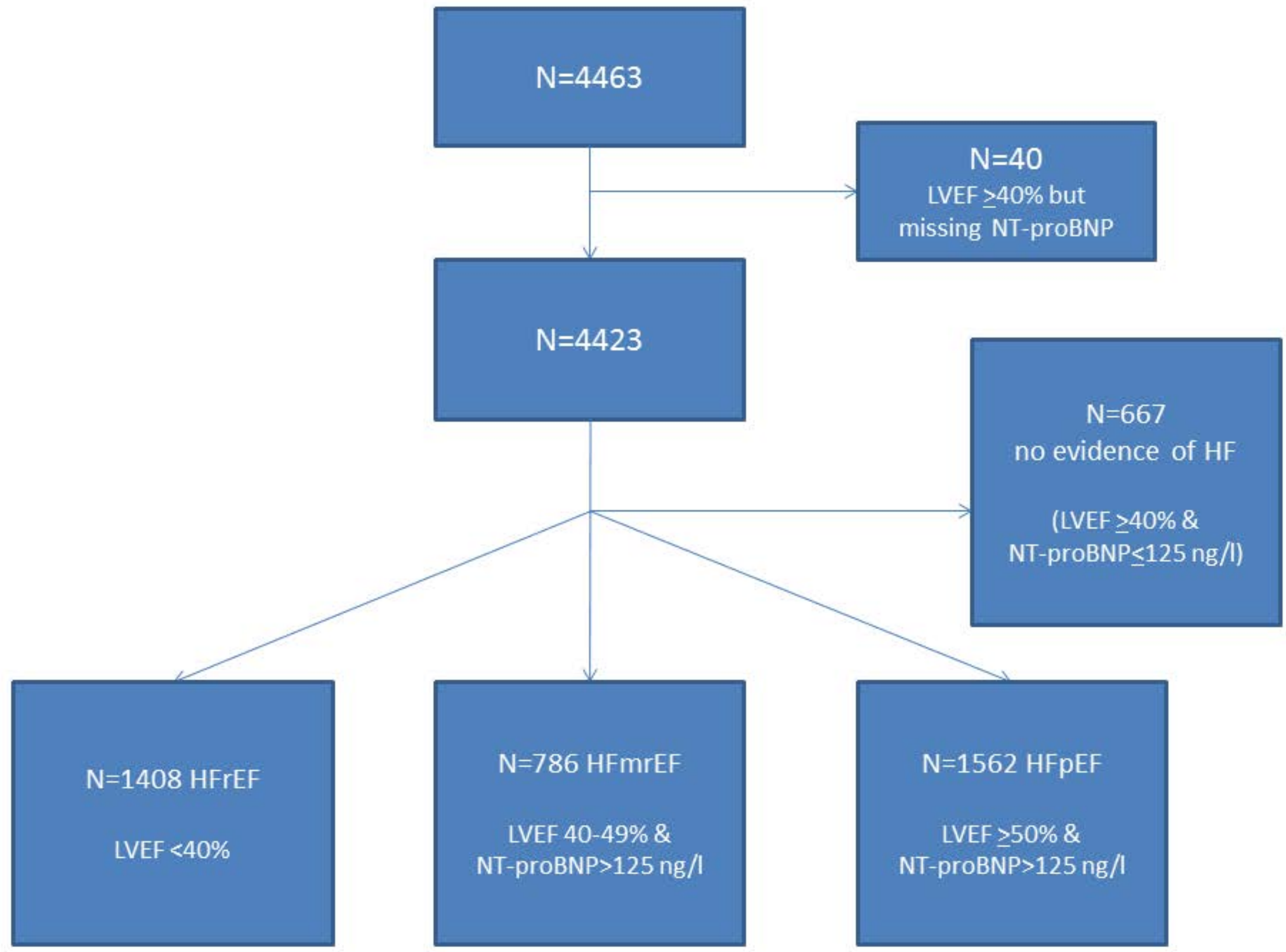

